\title{
Quantification of the natural convection perturbations on differential scanning calorimetry measurements of PCMs
}

\author{
Damien David ${ }^{\mathrm{a}, \mathrm{b}, *}$, Kevyn Johannes ${ }^{\mathrm{a}, \mathrm{b}}$, Frederic Kuznik ${ }^{\mathrm{a}, \mathrm{c}}$ \\ ${ }^{a}$ Univ Lyon, CNRS, INSA-Lyon, Université Claude Bernard Lyon 1, CETHIL UMR5008, F-69621, Villeurbanne, France \\ ${ }^{b}$ Universite Claude Bernard Lyon 1 \\ ${ }^{c}$ INSA de Lyon
}

\begin{abstract}
The paper investigates the effect of natural convection during differential scanning calorimetry measurements of phase change materials. We performed series of numerical simulations to characterize and quantify the perturbation on the measured thermogram. This perturbation becomes significant at the end of the fusion process. It manifests itself as an increase of the heat flux amplitude which accelerates the fusion process. Three correlations are built from the simulation results. The two first correlations quantify the amplitude of the heat flux deviation. The third correlation locates the time at which the perturbation occurs. The correlations are valid for large DSC capsules (around $1 \mathrm{ml}$ ), and high Prandtl materials (paraffins, salt hydrates, fatty acids). They enable to define the experimental conditions for which natural convection becomes negligible.
\end{abstract}

Keywords: Phase Change Materials, Differential Scanning Calorimetry, Natural Convection, Thermogram, Lattice Boltzmann model

\section{Nomenclature}

\begin{tabular}{|c|c|c|c|}
\hline \multicolumn{2}{|c|}{ Latin and Greek letters } & \multicolumn{2}{|c|}{ Dimensionless numbers } \\
\hline$A$ & outer surface of the sample & $B i$ & Biot number \\
\hline$b_{\infty}$ & temperature growth rate & Fo & Fourier number \\
\hline $\begin{array}{l}c_{p} \\
h_{s}\end{array}$ & $\begin{array}{l}\text { specific heat } \\
\text { boundary heat transfer coefficient }\end{array}$ & $\tilde{F} O_{\max }$ & $\begin{array}{l}\text { Relative position of the maxi- } \\
\text { mum convection perturbation }\end{array}$ \\
\hline$H$ & due to the sensor location & $\Gamma$ & shape factor \\
\hline $\begin{array}{l}\Pi \\
L\end{array}$ & reference length & $N u$ & Nusselt number \\
\hline$L_{f}$ & latent heat & $\Delta N u_{\max }$ & maximum convection perturba- \\
\hline $\begin{array}{l}Q_{\text {ref }} \\
Q_{S}\end{array}$ & reference heat flux & $(\Delta N u / N u)_{\max }$ & $\begin{array}{l}\text { tion amplitude } \\
\text { maximum relative convection } \\
\text { perturbation amplitude }\end{array}$ \\
\hline R & sample radus & $\operatorname{Pr}$ & Prandtl number \\
\hline $\begin{array}{l}\rho \\
t\end{array}$ & density & $R a$ & Rayleigh number \\
\hline$t$ & time & $R S$ & Rayleigh over Stefan number \\
\hline$I_{\infty}$ & $\begin{array}{l}\text { lemperature imposed by tne reg- } \\
\text { ulation }\end{array}$ & $S t$ & Stefan number \\
\hline
\end{tabular}

\section{Introduction}

Latent heat storage system use Phase Change Materials (PCMs) to store heat. The PCM characteristic which has the strongest impact on the dynamics of the storage system is the PCM enthalpy curve (Darkwa

\footnotetext{
*Corresponding author: damien.david@insa-lyon.fr
} 
and O'Callagan [1], Izquierdo-Barrientos et al. [2], Mathieu-Potvin and Gosselin [3], Arkar and Medved [4], Kuyznik et al. [5]). This curve describes the evolution of the material specific enthalpy $h$ as a function of the temperature $T$.

The most popular way to obtain an enthalpy curve is to perform a Differential Scanning Calorimetry (DSC) measurement. A sample of the material is subjected to a temperature ramp, and the time evolution of the heat flux around the sample is measured. The relation between the temperature and the heat flux is called thermogram.

It is usually recommended to use small capsules (around $10^{-3} \mathrm{ml}$ ) and low temperature scan speeds when performing DSC measurements. The idea behind those recommendations is to approach the homogeneous thermal state in the PCM sample (homogeneous temperature, and homogeneous liquid or solid state), and to limit the effects of natural convection and conduction in the liquid phase of the sample.

However, using a small capsule and a low temperature scan speed requires a rigorous measurement procedure to reduce measurement errors. Extremely low heat flux amplitudes are expected. Those amplitudes approach the limit of sensitivity of the DCS device. The calibration of the device should be flawless to avoid bias in the heat flux measurements. Since the mass of the PCM sample is very small, it should be measured with an appropriate weighting scale in a non perturbed atmosphere. The contact between the PCM sample and the furnace should not be spoiled by impurities. Otherwise, the sample might not be homogeneously heated, and there might be temperature gradients between the sensor locations and the sample surface. PCMs are not necessarily pure materials, they can be mixtures of pure substances. For a PCM mixture, the composition of the PCM in a small sample might not be representative of the composition of the whole PCM. Several samples are needed to statistically approach the behaviour of an average PCM composition.

The rigour which is required for a DSC measurement with small capsules an low temperature scan speed makes the measurement procedure tedious. We are forced to notice that this rigour is not always met. Lazaro et al. [6] and Castellon et al. [7] conducted benchmark measurements all over Europe with n-Octadecane. They highlighted considerable discrepancies on thermograms due to non-rigorous experimental protocols.

The reproducibility of the calorimetry measurements is improved by using calorimeters with large capsules. A large capsule is a capsule whose volume is approximatively equal to $1 \mathrm{ml}$. The measurement principle is the same: the sample is subjected to a ramp of external temperature, and the heat flux which passes through the PCM sample is measured. Those calorimeters are commonly used to measure the heat capacity of standard solid materials. They can provide very precise heat flux measurements, with a great repeatability.

The utilization of large capsule calorimeters to get PCM thermograms rises two major issues. The first issue is due to the diffusion of the heat within the sample. Let's imagine the phase change front is near the core of a large PCM sample. It releases some latent energy. The latent energy takes some time to reach the sample surface. When it reaches the surface, it is measured by the device. So the heat flux which is measured at the surface does not reflects what happens in the sample right at the measurement time. It reflects what happened in the sample in the past. The diffusion process within the PCM sample must be taken into account for a reasonable interpretation of the thermogram.

Actually, heat diffusion is not an issue which is specific to large capsule measurements. Dumas et al. [8] pointed out that every thermogram was distorted by heat diffusion effects. That's why PCM thermograms depend so much on the temperature scan speed, whatever the device used. Franquet et al. [9] and Gibout et al. [10] decided to develop a numerical tool to take into account the heat diffusion when interpreting thermograms. Their tool consists in an inverse method based on a heat diffusion model. It performs successive guesses of the PCM enthalpy curve, until the corresponding simulated thermogram converges toward the measured thermogram. With this tool, heat diffusion perturbations can be extracted from the thermograms.

The second issue is the natural convection flow in the liquid region. It is well known that natural convection accelerates the heat transfer between the phase front and the heat transfer fluid in PCM storage units. The same phenomenon might occur in large DSC samples. If the volume of the liquid region is too large, and if the thermal gradients are too high, the viscosity might not be sufficient to restrain the natural convection flow. The subsequent perturbation on the thermogram is the central issue tackled in the present paper. 
Through this study, we wanted to answer three questions:

- how does the natural convection flow disturb the thermogram?

- how can the perturbation of the flow be quantified?

- how can it be related to the DSC measurement conditions, such as capsule size, temperature scan speed, etc?

We present in this paper the results of series of numerical simulations performed to answer the three previous questions. The first section of the document is devoted to the presentation of the numerical model of the PCM. This model was designed to reproduce as close as possible all the heat transfer phenomena in the PCM sample. Then, we focus on the shape of the natural convection perturbation, and on the way to parametrize this perturbation.

The two next sections concern the design of a tool to quantify the natural convection pertubation. We propose three correlations: two correlations concern the perturbation amplitude, and one correlation concern the time location of this perturbation. Those correlations are expressed as functions of the PCM material properties and the DSC measurement conditions.

Finally, the last section of the paper illustrates the proper way to use the three correlations through an example.

\section{Numerical model of the PCM sample}

\subsection{Geometry of the model and boundary conditions}

The geometry of the simulated system is an ideal representation of a DSC sample (Fig.1, right). The sample volume is a perfect cylinder; there is no volume compression or expansion during the phase change. Given the symmetry of the system, we use an axis-symmetric model: only a half-section of the sample is simulated.

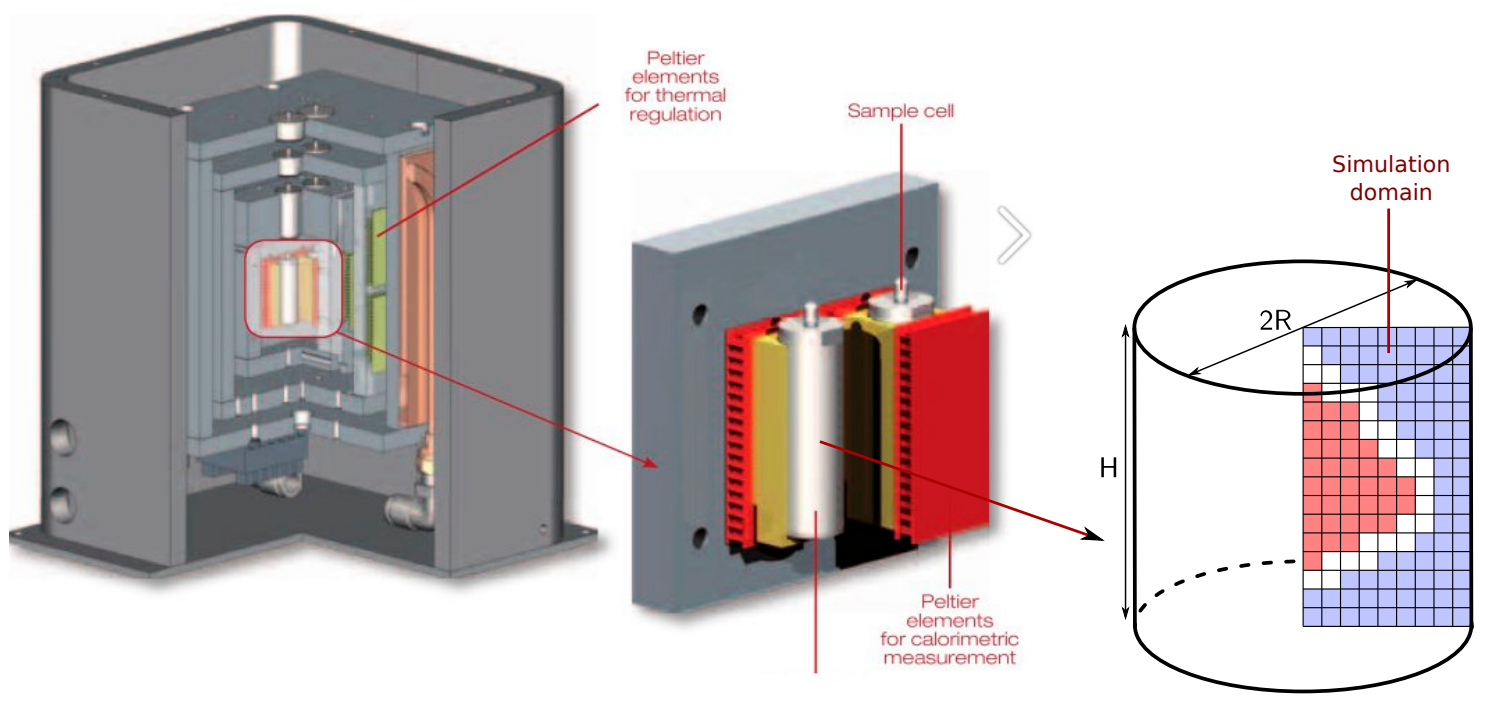

Figure 1: From the DSC calorimeter (left) to the sample simulation domain (right). (Initial picture from the commercial brochure of the calorimeter $\mu \mathrm{DSC} 7$ evo from Setaram)

The thermal boundary conditions are shown in Fig.2. The symmetry axis is adiabatic. DSC devices are designed to impose a ramp of temperature $T_{\infty}(t)$ on the sample: 


$$
T_{\infty}=T_{0}+b_{\infty} \times t
$$

An ideal DSC device would apply the temperature $T_{\infty}$ directly on the sample surface. However, the temperature sensor from which the temperature $T_{\infty}$ is regulated is never located exactly at the surface of the PCM sample. We model the heat transfer between the furnace location where $T_{\infty}$ is measured and the sample surface nodes through a heat transfer coefficient $h_{S} . h_{S}$ is homogeneous all over the sample surface. The boundary temperatures $T_{S}$ and heat flux densities $q_{S}$ are related to each other with mixed boundary conditions:

$$
q_{S}=h_{S}\left(T_{\infty}-T_{S}\right)
$$

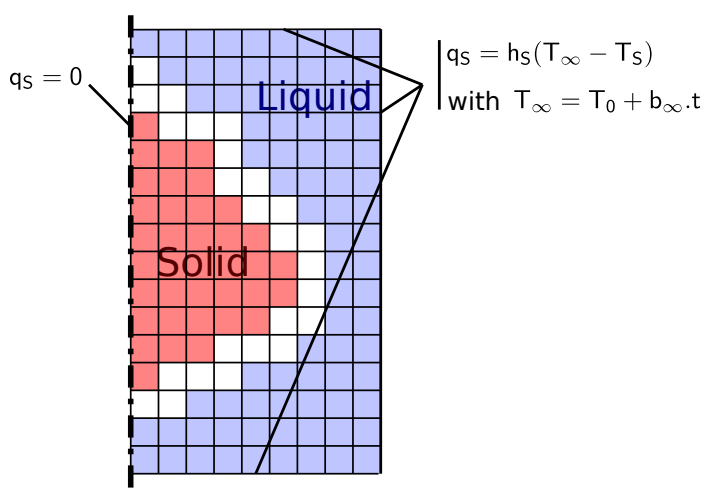

Figure 2: Boundary conditions for the energy conservation model

The boundary conditions of the fluid flow model are shown in Fig.3. The phase change material does not fill the entire volume of the DSC capsule. That's why we use a free surface boundary condition on the top of the simulation domain (non-zero radial velocity component). Non-slip boundary conditions are applied on the right and bottom boundaries, and on the solid-fluid interface. Axis-symmetric boundary conditions are applied on the axis boundary.

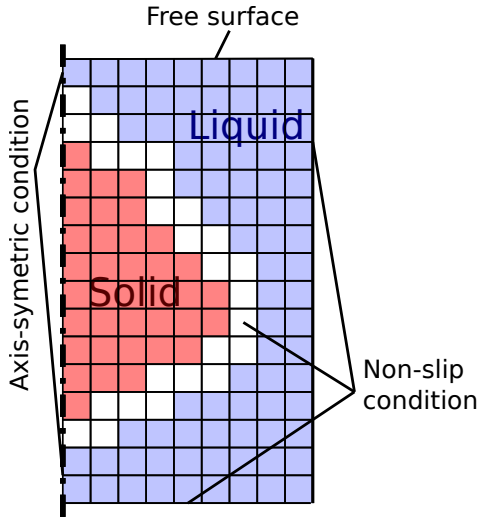

Figure 3: Boundary conditions for the fluid flow model

The simulated material is a pure PCM. Its enthalpy curve is shown in Fig. 4. The latent heat of the material is $L_{f}$, its fusion temperature is $T_{f}$. The specific heat $c_{p}$ is the same in the liquid and in the solid 
regions. The relation between the material specific enthalpy $h$, its temperature $T$ and its liquid fraction $l_{f}$ is:

$$
h=\left\{\begin{array}{l}
c_{p} T \quad \text { solid phase }\left(T<T_{f}\right) \\
c_{p} T_{f}+l_{f} L_{f} \quad \text { phase change }\left(T=T_{f}\right) \\
c_{p} T+L_{f} \quad \text { liquid phase }\left(T>T_{f}\right)
\end{array}\right.
$$

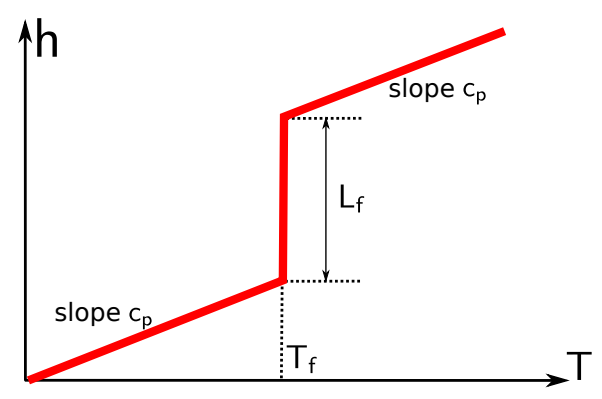

Figure 4: Enthalpy curve of the simulated PCM

The output of the simulation is the evolution of the heat flux $Q_{S}(t)$ which crosses the sample boundary. Since no convection exists during solidification, only fusion cycles are computed $\left(b_{\infty}>0\right)$.

\subsection{Fluid flow model}

The fluid flow model is activated only in the liquid region, where $l_{f}>0.5$. It is an axis-symmetric Lattice Boltzmann model, which is based on the model developed by Guo [11]. This is a D2Q9 model: two dimensions / nine particle velocities. The mesh is a square mesh. The width of each lattice is $\Delta X$. On each mesh node, the nine particle velocities join the node with itself and with the eight neighbouring nodes during one time step:

$$
\begin{aligned}
& \vec{c}_{0}=(0 ; 0) \\
& \vec{c}_{1-4}=( \pm 1 ; 0) \Delta X / \Delta t,(0 ; \pm 1) \Delta X / \Delta t \\
& \vec{c}_{5-8}=( \pm 1 ; \pm 1) \Delta X / \Delta t
\end{aligned}
$$

One distribution function $f_{i}$ is associated to each particle velocity. The distribution functions are related to the macroscopic density $\rho$, velocities $\vec{u}$ and forces $\vec{F}$ through their zero-th and first order moments:

$$
\begin{aligned}
& \sum_{i} f_{i}=r \rho \\
& \sum_{i} \vec{c}_{i} f_{i}=r \rho \vec{u}-\frac{\Delta t}{2} r \vec{F}
\end{aligned}
$$

The distribution functions follow a discrete evolution given by equation 6 . Each time step is divided into two sub steps: the streaming step (left hand side), the collision step (right hand side).

$$
f_{i}\left(\vec{x}+\vec{c}_{i} \Delta t, t+\Delta t\right)-f_{i}(\vec{x}, t)=\frac{1}{\tau}\left(f_{i}-f_{i}^{e q}\right)+\Delta t\left(1-\frac{1}{2 \tau}\right) R_{i}
$$

The equilibrium distribution function is: 


$$
f_{i}^{e q}=r \rho \omega_{i}\left(1+\frac{\vec{c}_{i} \cdot \vec{u}}{c_{S}^{2}}+\frac{\left(\vec{c}_{i} \cdot \vec{u}\right)^{2}}{2 c_{S}^{4}}-\frac{\vec{u} \cdot \vec{u}}{c_{S}^{2}}\right)
$$

Where $c_{S}^{2}=\Delta X^{2} /\left(3 \Delta t^{2}\right)$ is the speed of sound. The relaxation time $\tau$ depends on the fluid kinematic viscosity: $\nu=\Delta t c_{S}^{2}(\tau-0.5)$. The source terms $R_{i}$ are related to the macroscopic forces:

$$
R_{i}=r \omega_{i}\left[\frac{\vec{c}_{i}-\vec{u}}{c_{s}^{2}}+\frac{\vec{c}_{i} \cdot \vec{u}}{c_{s}^{4}} \vec{c}_{i}\right] \cdot \vec{F}
$$

The force $\vec{F}$ is the sum of the real force applied to the fluid $\vec{F}_{0}$, and a corrective term $\vec{F}^{\prime}$ which is needed by the Lattice Boltzmann scheme to recover the Navier Stokes equations in cylindrical coordinates. Here, $\vec{F}_{0}$ is the natural convection force. It is approximated by the Boussinesq formulation:

$$
\vec{F}_{0}=\rho g \beta\left(T-T_{r e f}\right) \vec{e}_{z}
$$

The corrective term is:

$$
\vec{F}^{\prime}=\left(\rho \frac{c_{S}^{2}}{r}-\rho \nu \frac{2 u_{r}}{r^{2}}\right) \vec{e}_{r}
$$

The no-slip boundary conditions on the walls and the solid-liquid interface are modelled with a bounceback scheme (Gallivan et al. [12]). The free surface is simulated with a mirror boundary condition technique (Kuo and Chen [13]). The symmetry condition on the axis is simulated with a specific scheme developed by David et al. [14].

\subsection{Energy conservation model}

The energy conservation model is based on the enthalpy method. For the sake of simplicity, the fluid model and the energy model share the same time an space meshes. The time scheme of the energy model is Euler explicit. The diffusion terms are discretized using the control volume technique from Patankar [15]. The convection terms are treated with a second order, centred finite difference scheme.

The simulation domain is split up into square control volumes. Let $\tilde{q}_{r-}, \tilde{q}_{r+}, \tilde{q}_{z-}$ and $\tilde{q}_{z+}$ be the estimations of the heat flux densities on the four sides of a control volume. Let $\partial_{r} T$ and $\partial_{z} T$ and be the estimations of the thermal gradients on the radial and vertical directions. The subscript + refers to the quantities at the time $t+\Delta t$, the absence of subscript corresponds to the quantities at the time $t$. We get the average specific enthalpy in the control volume $h^{+}$from the following equation:

$$
\rho h^{+}=\rho h+\Delta t\left[-\rho c_{p}\left(u_{r} \partial_{r} T+u_{z} \partial_{z} T\right)+\frac{\tilde{q}_{r-}+\tilde{q}_{r+}+\tilde{q}_{z-}+\tilde{q}_{z+}}{\Delta X}+\frac{\tilde{q}_{r+}+\tilde{q}_{r-}}{2 r \Delta X}\right]
$$

The average temperature $T^{+}$and the liquid fraction $l_{f}^{+}$are obtained from $h^{+}$by using the enthalpy curve of the material (Eq.3 and Fig.4) .

\subsection{Stability, precision of the model}

The three following criterion were fulfilled to ensure the stability of the energy conservation model (Pletcher et al. [16]):

- Diffusion criterion: $\alpha \Delta t / \Delta X^{2}<0.25$ 
- CFL criterion: $U_{0} \Delta t / \Delta X<0.5$

- Mixed criterion: $U_{0}^{2} \Delta t / \alpha=1$

The reference velocity is $U_{0}=\sqrt{q \beta \Delta T L}$. The consistency between the Lattice Boltzmann model and the Navier Stokes equations is ensured by setting $M a=U_{0} / c_{s}<0.1$, where $M a$ is the Mach number.

An optimal number of grid points was determined by a mesh dependency study for each capsule geometry. The studies were carried out for the most sensitive set of parameter: low Rayleigh and high Stefan numbers. The mesh was refined until we reached a stabilization of $\Delta N u_{\max }$ within $2 \%$ (The definition of $\Delta N u_{\max }$ is given below).

\section{Description of the convection perturbation}

In this section, we analyse one simulation result to describe the mechanism of natural convection in the PCM. The results present the fusion of Capric Acid (Table 2), in a $11 \mathrm{~mm}$ diameter and $11 \mathrm{~mm}$ height furnace, with a scanning speed $b_{\infty}=11 K / h$.

An analysis of the velocity streamlines is provided in the first part of the present section. This analysis reveals different stages in the formation of vertices in the liquid region. Their effect on the thermogram are described in the second part of this section, and some parameters which quantify the convection perturbation on the thermogram are defined in the last part.

\subsection{Flow patterns}

The Figure 5 shows four snapshots of the velocity streamlines during the melting process. At the beginning, the liquid area is too thin to enable a strong convective current. At this stage the natural convection should not disturb the heat flux evolution.

Then, as the fusion front moves on, it leaves enough space for two convection vertices: one at the top and one at the bottom corner of the furnace. The vertices are driven by the temperature difference between the fusion front (cold surface) and the furnace wall (hot surface). The more they grow, the stronger should be the convection perturbation.

After a moment, the two vertices merge into one single toroidal vortex. At this stage, the convection perturbation should be maximum. It accelerates the heat transfer between the solid PCM and the furnace wall. The last picture show that the fluid movement persists after the end of the phase change.
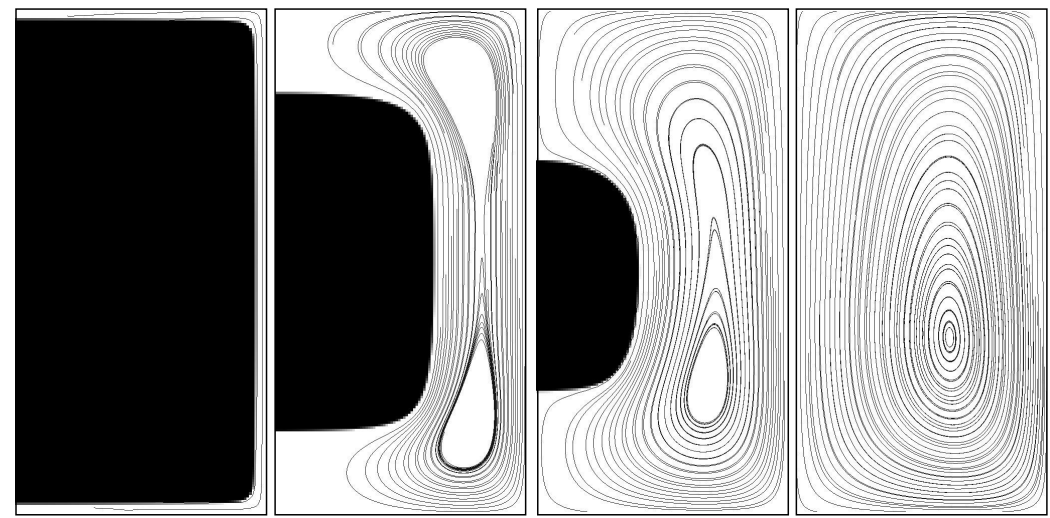

Figure 5: Evolution of the velocity streamlines in the liquid region during the melting process. The symmetry axis of the furnace is located on the left side of the pictures. The fluid is moves couter-clockwise. The solid region is the black area 


\subsection{Specific thermogram representation}

This paper presents the thermograms in a dimensionless form. The definition of dimensionless quantities involves a reference heat flux $Q_{r e f}$. The reference heat flux is the heat flux that we can expect when the PCM is fully solid or fully liquid. After some time, a stationary regime occurs. During the stationary regime, the temperature growth rate is homogeneous within all the PCM volume, it is equal to $b_{\infty}$. The expected heat flux is:

$$
Q_{\text {ref }}=V \rho c_{p} b_{\infty}=\pi R^{2} H \rho c_{p} b_{\infty}
$$

The thermograms are expressed in terms of Nusselt number $N u$ evolutions over the Fourier number $F o$. The Nusselt number is the dimensionless form of the measured heat flux $Q_{S}$ : it is the ratio between $Q_{S}$ and the reference heat flux $Q_{r e f}$. The Fourier number is the dimensionless form of the time $t$. It depends on the diffusivity $\alpha$ of the PCM, and a reference length which will be defined later in the paper:

- Nusselt number: $N u=\frac{Q_{S}}{Q_{r e f}}$

- Fourier number: $F_{O}=\frac{\alpha t}{L^{2}}$

Let's now have a look on Fig.6. For the moment, we focus on the simulation results without convection effect (dashed blue lines). The top figure contains the thermogram $N u_{C D}(F o)$. The middle figure contains the evolution of the global liquid fraction $l_{f}\left(F_{0}\right)$. The global liquid fraction is the fraction of the PCM volume which is occupied by the liquid region.

The fusion process causes an increase of the Nusselt number $N u$, which occurs between two stationary regimes: the solid state stationary regime $\left(F_{O} \leq 0\right)$, and the liquid state stationary regime $\left(F_{o} \geq 23\right)$. The Nusselt number is equal to 1 during both stationary regimes $\left(Q_{S}=Q_{r e f}\right)$. The line $N u=1$ is the base line of the calorimetry measurement.

The time scale was shifted so that $F_{o}=0$ when $T_{\infty}=T_{f}$. We notice that the rise of the heat flux does not start exactly when $F o=0$, but shortly after. This delay is due to the thermal resistance between the temperature $T_{\infty}$ and the PCM surface temperature.

The middle curve shows that $l_{f}=1$ from $F o \approx 17.3$. From this limit, the PCM is fully liquid (grey area), the fusion process is over. However, we still observe $N u>1$ after $F_{o} \approx 17.3$. This is due to the heat diffusion process inside the PCM sample.

\subsection{The shape of the convection perturbation}

The thermogram $N u_{C V}(F o)$ was obtained with the entire model (energy + fluid flow) (top of Fig.6). It represents the Nusselt evolution which is distorted by the natural convection flow. The convection perturbation is the difference between the curves $N u_{C V}(F o)$ and $N u_{C D}(F o)$.

Consequently, a new quantity called the absolute convection perturbation: $\Delta N u=N u_{C V}-N u_{C D}$ is defined. The evolution of $\Delta N u$ is shown in the bottom curve of Fig. 6. As expected, the convection perturbation is null at the beginning of the fusion $\left(F_{o}<10\right)$. At this stage, the natural convection flow is too weak to significantly increase the heat transfer rate of in the liquid region.

From $F o=10$ the perturbation gradually increases until it reaches a maximum value. We can't see on the perturbation evolution any accident that could be interpreted by the fusion of the two vertices.

Since the natural convection increases the heat transfer between the solid region and the furnace wall, the solidification process ends up earlier with the natural convection perturbation $\left(F_{O} \approx 17\right)$. The decrease of $\Delta N u$ starts shortly after. It continues until $\Delta N u$ reaches negative values. Negative values of $\Delta N u$ are due to the fact that the fusion process is longer without convection effect: after some time, we get $N u_{C D}>N u_{C V}$ 


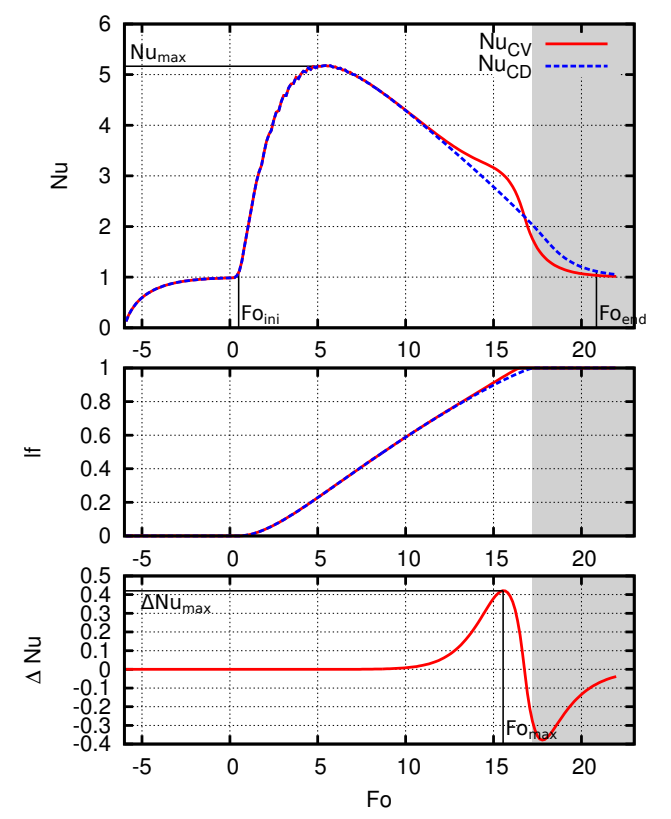

Figure 6: Typical result from one simulation. Conduction and conduction + convection thermograms in the top figure, evolutions of the global liquid fraction in the middle figure, convection perturbation in the bottom figure

\subsection{Quantification of the convection perturbation}

We defined three quantities to quantify the convection perturbation.

The first quantity is $\Delta N u_{\max }$. It reflects the amplitude of the convection perturbation. It is the maximum value of $\Delta N u$ during the whole fusion process. It is identified in the bottom curve of Fig.6. If the value of $\Delta N u_{\max }$ is equal to 1.5 , it means that the maximum value of the convection perturbation is equal to 1.5 times the value of the heat flux at the base line.

The second quantity is $(\Delta N u / N u)_{\max }$. It also reflects the amplitude of the convection perturbation, but in terms of instantaneous relative heat flux value. It is the maximum value of $\Delta N u / N u_{C D}$ during the whole fusion process.

The third quantity $\tilde{F}_{O_{\max }}$ reflects the stage of fusion process at which the maximum perturbation occurs. This parameter is important because the later the convection perturbation occurs, the lower is its influence on the inverse model which is used to retrieve the PCM enthalpy curve from the thermogram. Let $F_{o_{0}}$ and $\mathrm{Fo}_{\text {end }}$ be the values of the Fourier number at the beginning and the end of the phase change effect (Fig. 6). $F o_{0}$ occurs when $N u_{C D}$ has risen $5 \%$ of its way to the maximal value $N u_{\max }$ :

$$
N u_{C D}\left(F o_{0}\right)=1+0.05 \times\left(N u_{\max }-1\right)
$$

$F o_{\text {end }}$ occurs when $N u_{C D}$ has returned to the previously given value. The maximum value of $\Delta N u$ is reached when $F o=F o_{\max }$. The maximum perturbation location $\tilde{F}_{O_{\max }}$ is defined by equation 14. It is close to 1 when the convection effect occurs at the end of the fusion process, and 0 when convection occurs at the beginning.

$$
\tilde{F} o_{\max }=\frac{F o_{\max }-F o_{0}}{F o_{\text {end }}-F o_{0}}
$$




\section{Presentation of the parametric study}

\subsection{Methodology}

The rest of the paper is devoted to the construction of correlations for $\Delta N u_{\max },(\Delta N u / N u)_{\max }$, and $\tilde{F}_{O_{\max }}$ as functions of the DSC experimental conditions. Those correlations might be used by DSC users to quantify the convection perturbation they can expect on their thermogram, and adjust the PCM quantity to limit undesirable effects.

The first step of the methodology was to define a set of parameters which fully describe the conditions of a DSC measurement. Five parameters are sufficient. They are are expressed in terms of dimensionless numbers. They are defined in the first part of this section.

An interval of study was defined for each representative parameter. The union of those intervals constitutes the range of validity of the future correlations. The intervals of study were established from an inventory of available phase change material thermal properties and available characteristics DSC devices. The inventories are described in the second part of the present section.

We used the full map of experiment method to define the list of simulations to perform in order to get a sufficient amount of data to build the correlations. The full map of experiment consists in performing one simulation for each combination of extreme values of the representative parameters. For each simulation, each representative parameter is set either to its maximum value, or to its minimum value. A total of $2^{5}=32$ simulations were performed.

A sensitivity analysis was performed on the simulation results, to determine which representative parameter was the the most influential on the values of $\Delta N u_{\max },(\Delta N u / N u)_{\max }$, and $\tilde{F}_{O_{\max }}$. The results of the sensitivity analysis drove the determination of the correlation shapes. Finally, the correlation parameters were fitted to the simulations results. The simulation results and the different stages of the correlation design are presented in the next section.

\subsection{Definition of the representative parameters}

The dimensionless parameters depend on a characteristic length $L$ and a characteristic temperature difference $\Delta T$. The characteristic length is the ratio between the sample volume and its outer area:

$$
L=\frac{V}{A}=\frac{\pi R^{2} H}{2 \pi R H+2 \pi R^{2}}=\frac{R H}{2(R+H)}
$$

The characteristic temperature difference $\Delta T$ depends on the reference heat flux density $Q_{r}$ ef $/ A$ and on $L$

$$
\Delta T=\frac{Q_{r e f}}{A} \frac{L}{k}=\frac{V \rho c_{p} b_{\infty}}{A} \frac{L}{k}=\frac{L^{2} b_{\infty}}{\alpha}
$$

The governing equations of the system are the Navier-Stokes equations with the Boussinesq approximation, and the energy conservation equation. By turning those equations into their dimensionless formulation, five dimensionless parameters appear:

- Prandtl number: $\operatorname{Pr}=\frac{\nu}{\alpha}$

- Rayleigh number: $R a=\frac{g \beta \Delta T L^{3}}{\alpha \nu}$

- Stefan number: $S t=\frac{c_{p} \Delta T}{L_{f}}$

- Shape factor: $\Gamma=\frac{H}{R}$

- Biot number: $B i=\frac{h_{S} L}{k}$

The five dimensionless parameters are sufficient to describe entirely the conditions under which the scanning calorimetry simulation is performed. 


\subsection{Intervals of study}

\subsubsection{Inventory of DSC devices characteristics}

In this paper, we focus on calorimetry devices with a large furnace volume. We identified three appropriate products: the $\mu \mathrm{DSC7evo}$ calorimeter from SETARAM, the $C 80$ calorimeter from SETARAM, and the MMC274 Nexus calorimeter from NETZSCH. The characteristics of those devices are gathered in table 1. The values of the heat transfer coefficient $h_{S}$ were estimated by Gibout [17] with the inverse conduction model.

\begin{tabular}{l|l|l|l|l|l}
\hline Product & $R$ & $H$ & $b_{\infty}$ & $h_{S}$ & $\mathrm{~V}$ \\
& $m m$ & $m m$ & $K / h$ & $W / m^{2}$ & $\mathrm{ml}$ \\
\hline$\mu$ DSC7evo & 3.2 & 15 & {$[10-120]$} & {$[200-400]$} & 0.75 \\
$C 80$ & 7.5 & 50 & {$[1-120]$} & {$[200-400]$} & 8.8 \\
MMC274 Nexus & 5 & 35 & {$[1-120]$} & {$[200-400]$} & 2.74 \\
\hline
\end{tabular}

Table 1: Characteristics of scanning calorimetry devices with large furnace volumes

\subsubsection{Inventory of PCM thermal characteristics}

We selected 14 phase change materials to get a representative set of PCM thermophysical data. Those data were found in Hasan et al. [18], Su et al. [19] and Humphries and Griggs [20]. They are gathered in Table 2 .

\begin{tabular}{l|l|l|l|l|l|l|l|l}
\hline Type & Material Name & $\rho$ & $c_{p}$ & $k$ & $\alpha$ & $\nu$ & $\beta$ & $L_{f}$ \\
& & $k g / m^{3}$ & $J / k g K$ & $W / m K$ & $m^{2} / s$ & $m^{2} / s$ & $1 / K$ & $k J / K$ \\
\hline Paraffins & n-tridecane C13 & 795 & 1500 & 0.14 & $1.17 e^{-7}$ & $8.81 e^{-7}$ & $9.4 e^{-4}$ & 154 \\
{$[20]$} & n-hexadecane C16 & 810 & 1600 & 0.15 & $1.16 e^{-7}$ & $1.11 e^{-6}$ & $9.3 e^{-4}$ & 228 \\
& n-octadecane C18 & 820 & 1600 & 0.15 & $1.14 e^{-7}$ & $1.34 e^{-6}$ & $9.2 e^{-4}$ & 244 \\
& n-Tetracosane C24 & 830 & 2300 & 0.15 & $7.86 e^{-8}$ & $1.57 e^{-6}$ & $8.5 e^{-4}$ & 162 \\
\hline Fatty & Capric Acid & 878 & 2200 & 0.15 & $7.92 e^{-8}$ & $7.21 e^{-6}$ & $7.45 e^{-4}$ & 153 \\
Acids & & & & & & & & \\
{$[19]$} & PEG 900 & 1150 & 2260 & 0.19 & $7.23 e^{-8}$ & $6.09 e^{-6}$ & $7^{-4}$ & 150 \\
& Stearic Acid & 900 & 2000 & 0.17 & $9.61 e^{-8}$ & $8.12 e^{-6}$ & $6.08 e^{-4}$ & 212 \\
& Erytritol & 1380 & 2300 & 0.5 & $1.58 e^{-7}$ & $5.07 e^{-6}$ & $7 e^{-4}$ & 340 \\
\hline Hydrates & Sodium Sulphate $+12 \mathrm{H}_{2} 0$ & 1845 & 2000 & 0.54 & $1.83 e^{-7}$ & $1.14 e^{-6}$ & $5 e^{-4}$ & 251 \\
{$[19]$} & Calcium Chloride $+12 \mathrm{H}_{2} 0$ & 1710 & 1900 & 0.75 & $2.31 e^{-7}$ & $1.14 e^{-6}$ & $5{ }^{-4}$ & 180 \\
& Magnesium Chloride $+6 \mathrm{H}_{2} \mathrm{O}$ & 1500 & 2500 & 0.6 & $1.6 e^{-7}$ & $1.14 e^{-6}$ & $5 e^{-4}$ & 167 \\
& Calcium Chloride $+6 \mathrm{H}_{2} \mathrm{O}$ & 1620 & 1830 & 0.75 & $2.53 e^{-7}$ & $1.14 e^{-6}$ & $5 e^{-4}$ & 212 \\
\hline Eutectics & CL & 830 & 2050 & 0.14 & $8.29 e^{-8}$ & $2.2 e^{-6}$ & $6.7 e^{-4}$ & 188 \\
{$[18]$} & CP & 840 & 2300 & 0.14 & $7.30 e^{-8}$ & $2.3 e^{-6}$ & $7.8^{-4}$ & 195 \\
\hline
\end{tabular}

Table 2: Thermal and physical properties of phase change materials

\subsubsection{Intervals of study}

The minimum and the maximum values of the dimensionless numbers were computed from tables 1 and 2. They are listed in Table 3. They form the interval of study of each parameter.

It is not correct to directly plan our simulations based on the five dimensionless parameters: $S t, R a, \Gamma$, $\operatorname{Pr}$ and $B i$. In that case, half of the simulations would represent unrealistic conditions. This is because both parameters $R a$ and $S t$ are proportional to $b_{\infty}$. The space occupied by the values of $R a$ and $S t$ is represented in Fig.7. We never get a maximum value of $R a$ when $S t$ is minimal. Similarly, we never get a minimum value of $R a$ when $S t$ is maximum.

To avoid non-physical simulation conditions, we replace $R a$ by a new parameter called $R S . R S$ is the ratio between $R a$ and $S t$ (Eq.17). It does not depend on $b_{\infty}$. The space occupied by $R S$ and $S t$ is represented in Fig.8. It has a rectangle shape with four physical corners. 


\begin{tabular}{ll|l|l}
\hline & & $\min$ & $\max$ \\
\hline$L$ & $m$ & $1.5 e^{-3}$ & $3.3 e^{-3}$ \\
$\Delta T$ & $K$ & 0.003 & 4.9 \\
$\Gamma$ & & 2 & 7 \\
$P r$ & & 10 & 100 \\
$R a$ & & 0.1 & 10000 \\
$S t$ & & $1 e^{-5}$ & 0.1 \\
$B i$ & & 2 & 4 \\
$R S$ & & 3000 & $3.5 e^{5}$ \\
\hline
\end{tabular}

Table 3: Intervals of study of the non dimension numbers

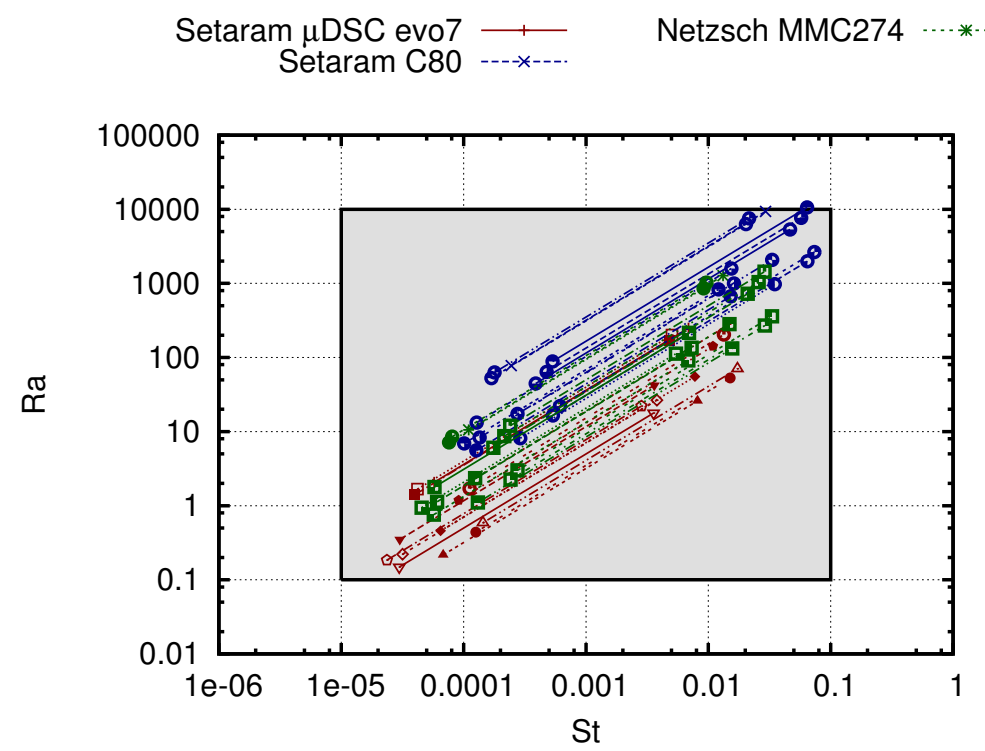

Figure 7: Surface occupied by the values of $R a$ and $S t$. The space occupied by the extremum values of $S t$ and $R a$ is represented with a grey rectangle. It contains two non physical extremal points located on the top left and on the bottom right corners.

$$
R S=\frac{R a}{S t}=\frac{\frac{g \beta \Delta T L^{3}}{\alpha \nu}}{\frac{c_{p} \Delta T}{L_{f}}}=\frac{g \beta L^{3} c_{p}}{L_{f} \alpha \nu}
$$

The interval of study of $R S$ is given in Tab.3. The simulation settings will be based on the intervals of study of the following five non dimension parameters: $S t, R S, \Gamma, P r$ and $B i$.

\section{Results of the parametric study}

\subsection{Results of the simulation campaign}

The simulated values of $(\Delta N u / N u)_{\max }, \Delta N u_{\max }$, and $\tilde{F}_{O_{\max }}$ are represented graphically in Fig.9. The combination of parameters of each simulation is indicated under the lower axis (+ for maximum value, for minimum value).

The relative perturbation amplitude $(\Delta N u / N u)_{\max }$ ranges from $0.02 \%$ (Simulation 7 ) to $100 \%$ (simulation 26). The perturbation amplitude ranges from 0.02 (simulation 6), to 60 (simulation 20). The relative 


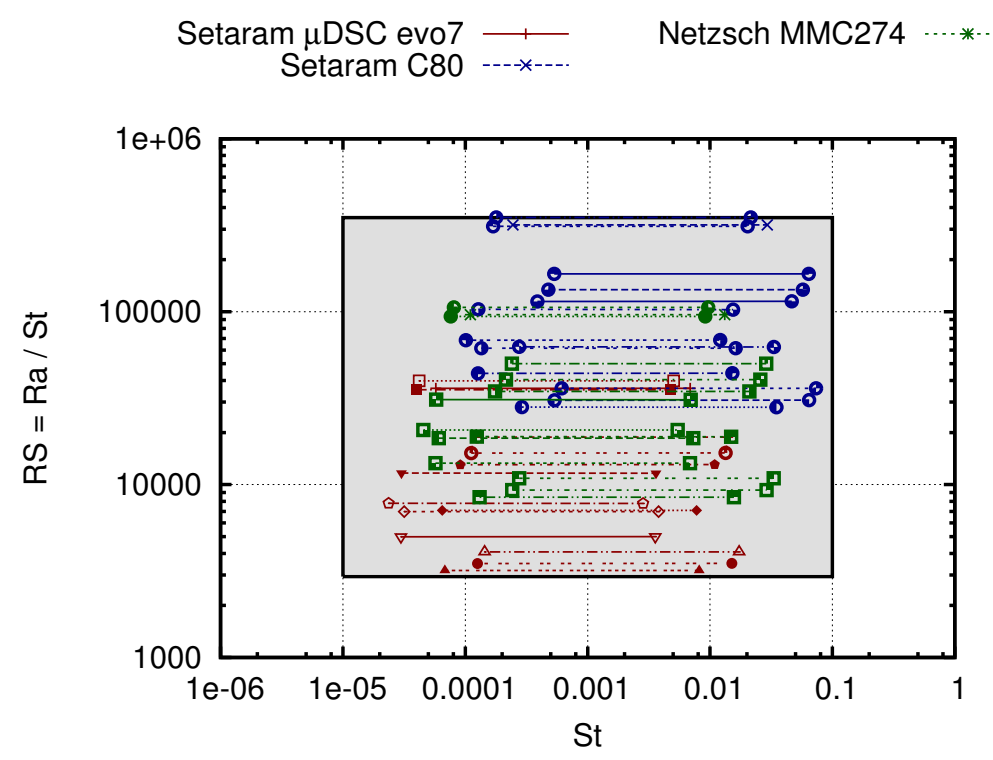

Figure 8: Space occupied by the values of $R S=R a / S t$ and $S t$. The resulting rectangle of study is drawn in grey. It does not have non physical corners

Fourier values range between $47 \%$ and $91 \%$ : the convection perturbation occurs mostly during the second half of the fusion process.

It is clear from Fig.9 that the parameters which have the strongest influence on the convection perturbation are the Stefan number $S t$ and the parameter $R S$. A calorimetry measurement with a low value of $S t$ is either the calorimetry measurement of a PCM which has a high latent heat $L_{f}$, or a calorimetry measurement with a very low scan speed $b_{\infty}$. For a given value of $S t$, the parameter $R S$ reflects the sensitivity of the fluid to thermal expansion.

Simulations 1 to 8 are the simulations with low $S t$ and low $R S$. They are the only simulations with a maximum relative perturbation below $10 \%$. For those simulations, the maximum perturbation occurs at the very end of the fusion process, corresponding to the physics of the heat transfer with natural convection.

The remaining simulations show stronger perturbations. The thermograms of simulations 1,917 and 25 are drawn in Fig.10. Those four simulations correspond to four different combinations of $[S t ; R S]$. The other parameter values are fixed. The perturbation is negligible in simulation 1 , because of the low values of $R S$ and $S t$.

When $S t$ (or $b_{\infty}$ ) increases (simulation 1 to 9 ), the baseline heat flux gets stronger compared to the heat flux due to the phase change. Thus, the Nusselt reaches smaller values during the phase change. In these conditions, the thermogram becomes more sensitive to the convection perturbation, which is responsible of a distinct second peak. The resulting thermogram is very similar to the thermogram of a binary mixture. It could lead to a misinterpretation of the curve.

Increasing $R S$ obviously increases the absolute perturbation amplitude $\Delta N u_{\max }$. But this increase is much more pronounced when $S t$ is low (simulation 1 to 17 ) than when $S t$ is higher. It is clear from Figs.9 and 10 that any modification of $S t$ and $R S$ which would lead to an increase of the convection perturbation, would also lead to a decrease of $\tilde{F}_{O_{\max }}$. The stronger is the perturbation, the sooner it occurs. 

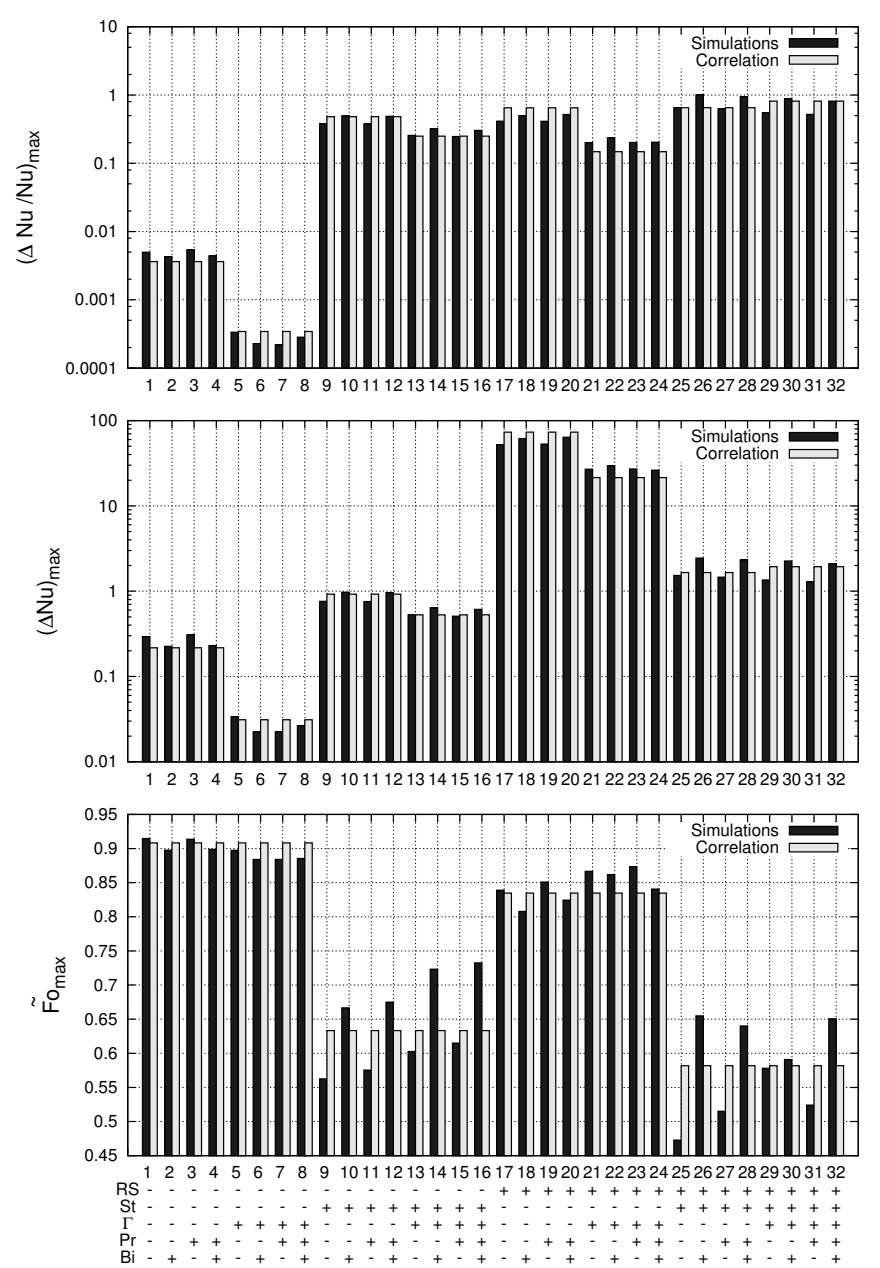

Figure 9: Results of the 32 simulations of the parametric study. For each simulation, the corresponding set of parameter is indicated under the lower axis. Comparison with the correlations predictions. 

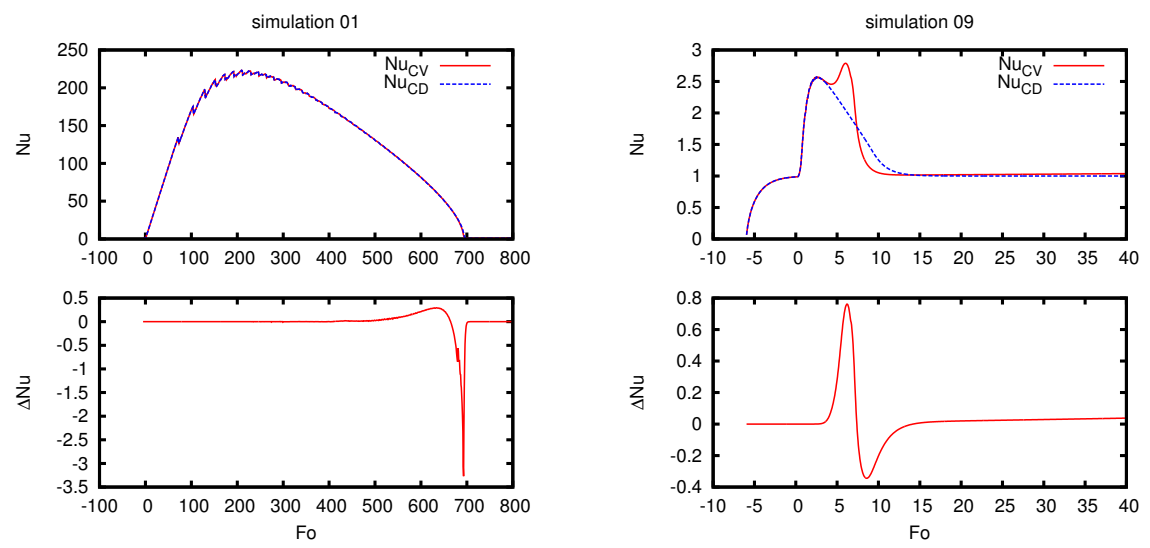

(a) Low RS, Low St

(b) Low RS, High St
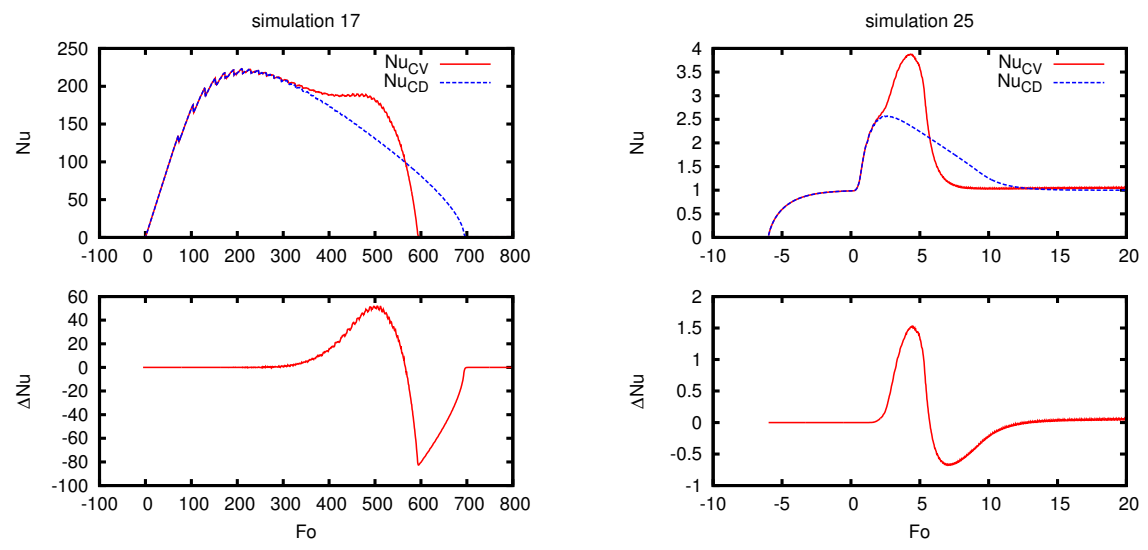

(c) High RS, Low St

(d) High RS, High St

Figure 10: Nusselt evolutions for simulations $0,8,16$ and 24 
The shape factor $\Gamma$ also influences the convection perturbation amplitude. A high value of $\Gamma$ results in a lower perturbation value, this is especially true when $S t$ and $R S$ are low. Since the top surface of the sample is free, the convection movement is not slowed down at this location. A high value of $\Gamma$ reduces the proportion of free surface at the top. It increases the contact with the walls, which damps the convection flow.

The Prandtl number and the Biot number have only a little effect on the convection perturbation amplitudes.

\subsection{The shape of the correlations}

Initially, we intended to build correlations by using the formalism given in equation 18. $Q$ is the quantity to be correlated: either $\Delta N u_{\max },(\Delta N u / N u)_{\max }$ or $\tilde{F} o_{\max }$. The coefficients $C_{0}, C_{R A}, C_{P r}, C_{S t}$ and $C_{B i}$ were obtained with a least square method.

$$
Q=C_{0} R a^{C_{R a}} \operatorname{Pr}^{C_{P r}} S t^{C_{S t}} \Gamma^{C_{\Gamma}} B i^{C_{B i}}
$$

We obtained a poor agreement between the simulation results and the correlation results. This is because the formalism of equation 18 does take into account the interactions between the system parameters. However, some interactions were highlighted in the previous section: the effect of $\Gamma$ on the perturbation amplitude is stronger when $S t$ and $R S$ (and $R a$ ) are small, the effect of $R S$ is much lower when $S t$ is low than when it is high. Moreover, the parameters $\operatorname{Pr}$ and $B i$ have a negligible influence on the simulation results. For those reasons, we decided to perform a sensitivity analysis to determine a more adequate formalism for the correlations.

The equation 18 is turned into a linear equation by applying the natural logarithm on each side:

$$
\ln (Q)=\ln \left(C_{0}\right)+C_{R a} \ln (R a)+C_{P r} \ln (P r)+C_{S t} \ln (S t)+C_{\Gamma} \ln (\Gamma)+C_{B i} \ln (B i)
$$

Then, the logarithm values are normalized to get values between -1 and 1 :

$$
\overline{\ln }(R a)=2 \times \frac{\ln (R a)-\ln \left(R a_{\min }\right)}{\ln \left(R a_{\max }\right)-\ln \left(R a_{\min }\right)}-1
$$

An the interactions are added to equation 20

$$
\ln (Q)=\overline{\ln }\left(C_{0}\right)+\bar{C}_{R a} \overline{\ln }(R a)+\bar{C}_{P r} \overline{\ln }(P r)+\bar{C}_{S t} \overline{\ln }(S t)+\bar{C}_{\Gamma} \overline{\ln }(\Gamma)+\bar{C}_{B i} \overline{\ln }(B i)+\text { interactions }
$$

The interactions are the products of all the possible combinations of $\overline{\ln }(R a), \overline{\ln }(P r), \overline{\ln }(S t), \overline{\ln }(\Gamma)$, and $\overline{\ln }(B i)$, multiplied by a corresponding coefficients. The 32 coefficients values are obtained by solving a linear system using the 32 simulation results. The resulting coefficient values are displayed in Fig.11.

The values of the sensitivity coefficient for $\Delta N u_{\max }$ and $(\Delta N u / N u)_{\max }$ are displayed in the top curve and the middle curve. As expected the coefficients for $\operatorname{Pr}$ and $B i$ are very low. We highlight two significant interactions: the interaction between $R a$ and $S t$, and the interaction between $R a$ and $\Gamma$. Despite the small value of $\bar{C}_{\Gamma}$, the term in $\Gamma$ will remain in the final form of the correlation to get a better resolution when $R a$ and $S t$ are small. We obtain the following formalism for $\Delta N u_{\max }$ and $(\Delta N u / N u)_{\max }$ :

$$
\ln (Q)=\ln \left(C_{0}\right)+C_{R a} \ln (R a)+C_{S t} \ln (S t)+C_{\Gamma} \ln (\Gamma)+C_{R a S t} \ln (R a) \ln (S t)+C_{R a \Gamma} \ln (R a) \ln (\Gamma)
$$

The bottom curve concerns $\tilde{F}_{O_{\max }}$. Here, we only keep the terms in $R a$ and $S t$ to get a reasonable approximation of the maximum perturbation location:

$$
\ln (Q)=\ln \left(C_{0}\right)+C_{R a} \ln (R a)+C_{S t} \ln (S t)
$$



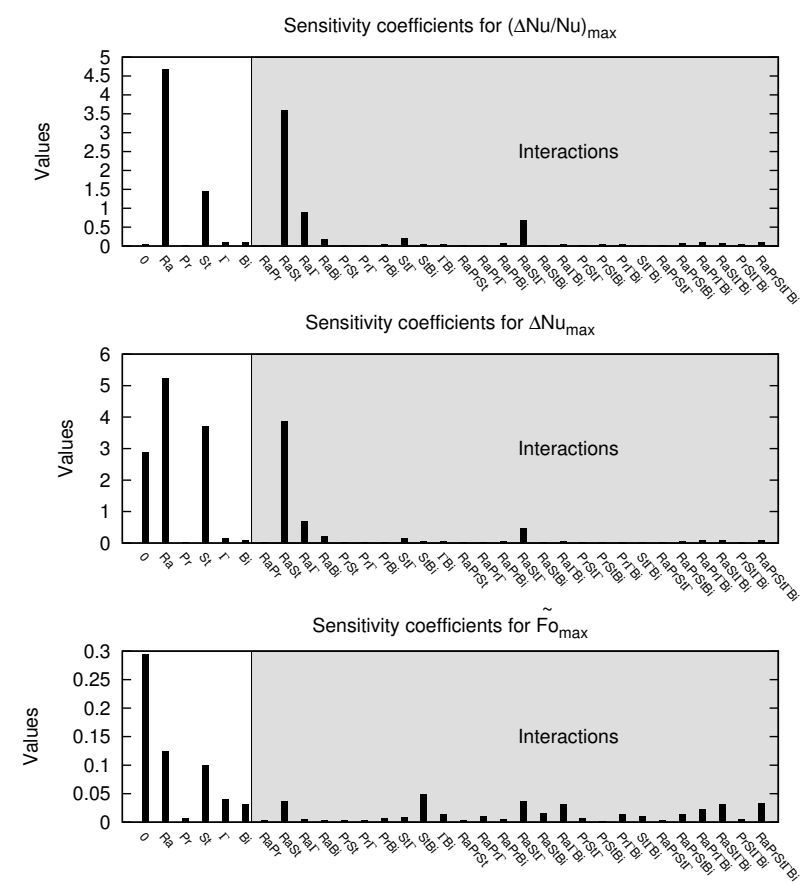

Figure 11: Sensibility of the non dimension parameters on the perturbation amplitudes and location. Effect of the coupling parameters

\subsection{Resulting correlations}

We built the correlations 24 and 25 and 26 by using the formalisms given in equation 22 and 23 , and by computing the coefficients with a least square method.

$$
\begin{aligned}
\Delta N u_{\max } & =0.41 \cdot S t^{-0.38} \cdot \Gamma^{-1.13} \cdot R a^{[-0.23-0.12 \ln (S t)+0.12 \ln (\Gamma)]} \\
\left(\frac{\Delta N u}{N u}\right)_{\max } & =1.03 \cdot S t^{0.077} \cdot \Gamma^{-1.37} \cdot R a^{[-0.3-0.11 \ln (S t)+0.15 \ln (\Gamma)]} \\
\tilde{F}_{O_{\max }} & =0.67 \cdot S t^{-0.021} \cdot R a^{-0.018}
\end{aligned}
$$

The correlation values are confronted to the simulation results in Fig 9. There is a good agreement between the correlation and the simulation values for the extremal values of the system parameters.

\section{How to use the correlations}

In this section, we illustrate how to use the correlation through an example. The PCM which is tested is a salt hydrate. Its latent heat is known from a previous measurement. It is equal to $180 \mathrm{~kJ} / \mathrm{kg}$. The objective is to determine its enthalpy curve by using a MMC274 Nexus calorimeter from NETZSCH. The constraint is less than $10 \%$ of perturbation due to natural convection on the thermogram.

The correlations enable us to calculate the amplitudes and the location of the convection perturbation as a function of the temperature scan speed $b_{\infty}$. This is done in Fig.12. If some PCM thermal properties are missing, we can select the thermal properties of similar materials in table 2 to compute the non dimension parameters. Here we selected the properties of the Calcium Choride $+12 \mathrm{H}_{2} \mathrm{O}$.

Since we want to limit the amplitude of the natural convection perturbation to $10 \%$, we need to set $(\Delta N u / N u)_{\max }<0.1$. This limit corresponds to a temperature scan speed which is equal to $18 \mathrm{~K} / \mathrm{h}(\mathrm{Fig} .12$, 

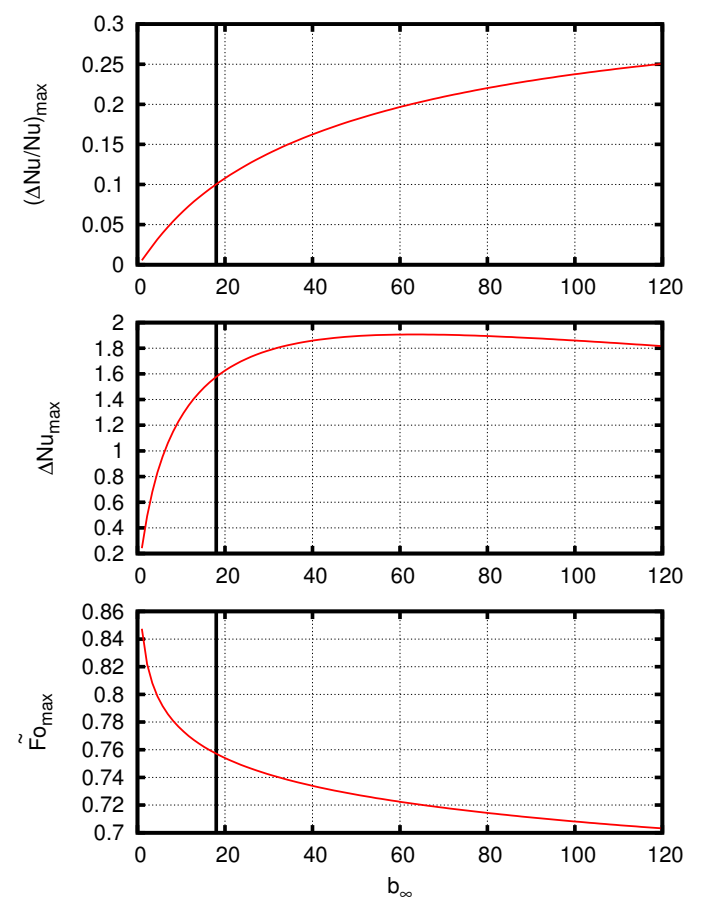

Figure 12: Example of how to use the correlation. Prediction of the natural convection perturbation amplitudes and location as a function of the temperature scan speed. The temperature scan speed $b_{\infty}$ is expressed in $K / h$

top curve). For that speed, we can expect the maximum perturbation to occur at $75 \%$ of the fusion process, with a value equal to 1.6 times the base line heat flux.

This configuration was simulated. The resulting thermogram is given in Fig.13. Here, the base line heat flux is $55.6 \mathrm{~mW}$. Since the correlation gave $\Delta N u_{\max }=1.6$ (Fig.12, middle curve), the maximum natural convection perturbation value should be $1.6 \times 55.6=88.96 \mathrm{~mW}$. This perturbation should occur at $75 \%$ of the fusion process (Fig.12, bottom curve).

The expected perturbation is drawn on the thermogram in Fig.13. The correlation slightly overestimates the perturbation amplitude. This could have been expected, given the concavity of the curves $(\Delta N u / N u)_{\max }\left(b_{\infty}\right)$ and $\Delta N u_{\max }\left(b_{\infty}\right)$. This overestimation is not an obstacle when we want to limit the amplitude of the perturbation.

\section{Conclusions}

In this paper, the effect of natural convection on DSC thermograms was quantified through series of simulations. The intervals of study were built around the case of DSC with large capsules $(\approx 1 \mathrm{ml})$, for the characterization of organic PCMs and Salt Hydrates (PCMs with high Prandtl values).

The results of the simulation campaign are aggregated in three correlations. They predict the maximum amplitude, the maximum relative amplitude and the time location of the maximum convection perturbation. The authors are aware of the limits of the model on which the correlation was built (non-moving solid phase in the capsule, homogeneous value of $h_{S}$ around the PCM, axis-symmetric system, etc). They are also aware of the tendency of the correlations to overestimate the perturbation amplitudes. Nevertheless, those correlations remain the first tool from which we can evaluate whether or not the natural convection would spoil the results of calorimetry measurement. 


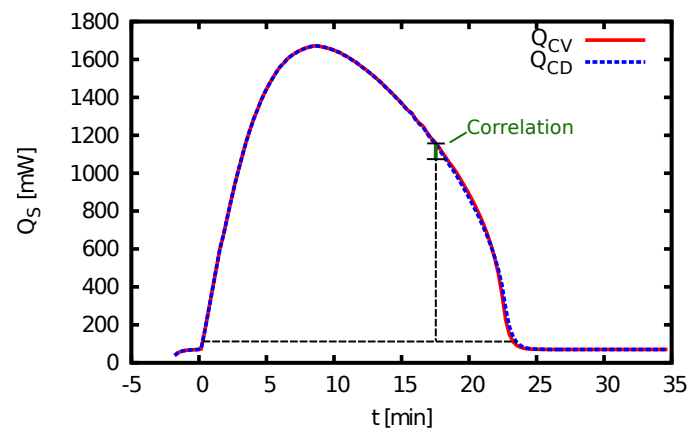

Figure 13: Example of how to use the correlation: Location of the convection perturbation on the thermogram

The correlations will be refined in a future work, by focusing on cases where $(\Delta N u / N u)_{\max }<0.2$.

From the simulation results, it is clear that small capsules are preferable to get low convection effect. However, small capsules require special care for the sample preparation, which may be a source of measurement errors. If one prefer bigger sample size, he should select narrow capsules and then adjust the temperature scan speed to reach acceptable convection effect.

\section{Acknowledgments}

This study was funded by the ANR project Stock-E MICMCP.

\section{References}

[1] K. Darkwa and P.W. O'Callaghan. Simulation of phase change drywalls in a passive solar building. Applied Thermal Engineering, 26(8-9):853-858, 2006.

[2] M.A. Izquierdo-Barrientos, J.F. Belmonte, D. Rodríguez-Sánchez, A.E. Molina, and J.A. Almendros-Ibáñez. A numerical study of external building walls containing phase change materials (pcm). Applied Thermal Engineering, 47(0):73 - 85, 2012.

[3] François Mathieu-Potvin and Louis Gosselin. Thermal shielding of multilayer walls with phase change materials under different transient boundary conditions. International Journal of Thermal Sciences, 48(9):1707 - 1717, 2009.

[4] C. Arkar and S. Medved. Influence of accuracy of thermal property data of phase change material on the result of a numerical model of packed bed latent heat storage with spheres. Thermochimica Acta, 438:192-201, 2005.

[5] F. Kuznik, K. Johannes, E. Franquet, L. Zalewski, S. Gibout, P. Tittelein, J. P. Dumas, D. David, J. P. Bedecarrats, and S. Lassue. Impact of the enthalpy function on the simulation of a building with phase change material wall. Energy and Buildings, 126:220-229, August 2016.

[6] Ana Lazaro, Conchita Peñalosa, Aran Solé, Gonzalo Diarce, Thomas Haussmann, Magali Fois, Belén Zalba, Stefan Gshwander, and Luisa F. Cabeza. Intercomparative tests on phase change materials characterisation with differential scanning calorimeter. Applied Energy, 109(0):415 - 420, 2013.

[7] C. Castellón, E. Günther, H. Mehling, S. Hiebler, and L. F. Cabeza. Determination of the enthalpy of pcm as a function of temperature using a heat-flux dsc - a study of different measurement procedures and their accuracy. International Journal of Energy Research, 32(13):1258-1265, 2008.

[8] Jean Pierre Dumas, Stéphane Gibout, Pierre Cézac, Erwin Franquet, and Didier Haillot. Model for the DSC thermograms of the melting of ideal binary solutions. Thermochimica Acta, 571(0):64 - 76, 2013.

[9] E. Franquet, S. Gibout, J.-P. Bédécarrats, D. Haillot, and J.-P. Dumas. Inverse method for the identification of the enthalpy of phase change materials from calorimetry experiments. Thermochimica Acta, 546(0):61 - 80, 2012.

[10] Stéphane Gibout, Erwin Franquet, William Maréchal, and Jean-Pierre Dumas. On the use of a reduced model for the simulation of melting of solutions in dsc experiments. Thermochimica Acta, 566(0):118 - 123, 2013.

[11] Zhaoli Guo, Haifeng Han, Baochang Shi, and Chuguang Zheng. Theory of the lattice boltzmann equation: Lattice boltzmann model for axisymmetric flows. Phys. Rev. E, 79:046708, Apr 2009.

[12] M.A. Gallivan, D.R. Noble, Georgiadis J.G., and R.O. Buckius. An evaluation of the bounce-back boundary condition for lattice boltzmann simulations. International Journal for Numerical Methods in Fluids, 25:249-263, 1997.

[13] Long-Sheng Kuo and Ping-Hei Chen. Numerical implementation of thermal boundary conditions in the lattice boltzmann method. International Journal of Heat and Mass Transfer, 52(1-2):529 - 532, 2009. 
[14] Damien David, Frederic Kuznik, Kevyn Johannes, and Lucie Merlier. Numerical analysis of truncation error, consistency, and axis boundary condition for axis-symmetric flow simulations via the radius weighted lattice Boltzmann model. Computers \& Fluids, 116:46-59, August 2015.

[15] S.V. Patankar. Numerical heat transfer and fluid flow. Taylor \& Francis, 1980.

[16] John C. Tannehill, Dale Dale Arden Anderson, and Richard H. Pletcher. Computational Fluid Mechanics and Heat Transfer, Second Edition. Taylor \& Francis, 1997.

[17] S. Gibout. Discussion with S. Gibout. 24th June 2013.

[18] A. Hasan, A, S. J. McCormack, M. J. Huang, and B. Norton. Phase change materials for thermal regulation of building integrated photovoltaics. PhD thesis, Doctoral Thesis. Dublin Institute of Technology, 2010.

[19] Weiguang Su, Jo Darkwa, and Georgios Kokogiannakis. Review of solid liquid phase change materials and their encapsulation technologies. Renewable and Sustainable Energy Reviews, 48:373-391, August 2015.

[20] W. R. Humphries and E. I. Griggs. A design handbook for phase change thermal control and energy storage devices. Technical report, November 1977. 